EUROPEAN ORGANIZATION FOR NUCLEAR REASEARCH

$P S-C D / 78-20$

STATUS REPORT ON CERN SC

by

B.W. Allardyce, A. Fiebig, G. Le Dallic, J.H.B. Madsen, P.H. Standley

Contribution to the

8th International Conference on Cyclotron and their Applications

Bloomington, USA

September 18-21, 1978

Geneva, Switzerland

September, 1978 



\section{STATUS REPORT ON CERN SC}

\section{B.W. Allardyce, A. Fiebig, G. Le Dallic, J.H.B. Madsen, P.H. Standley $\left.{ }^{\star}\right)$}

\section{Abstract}

The reconstructed CERN $600 \mathrm{MeV}$ Synchro-Cyclotron has been in operation since 1975. Down time in 1977 has been below 5\%; over two hundred experimental physicists use the machine (about half working with the Isotope on line Separator, Isolde). The bean is normally shared between an internal and an external target. Good macro duty cycle with virtually no $R F$ structure is obtained using a programmed dipole.

Since the spring 178 shutdown, an extracted ${ }^{3} \mathrm{He}^{2+}$ beam ( $910 \mathrm{MeV})$ is available and is being used both by Isolde and for counter experiments.

Project studies have shown the feasibility of accelerating ${ }^{12} \mathrm{C}^{4+}$ to $86 \mathrm{MeV} /$ nucleon (and other ions having $Q / A \simeq 1 / 3)$; authorization was received in June and the beam is planned for autumn 1979 .

\section{Introduction}

The performance of the CERN $600 \mathrm{MeV}$ Synchro-Cyclom tron (SC) has been considerably improved over the past three years with the gradual el inination of the teething troubles experienced after the SC Improvement Programme ${ }^{\star \star}$. The decision taken in summer 1976 , following the serious difficulties with the rotating condensers, to reduce the intensity goal from $10 u A$ to 4 A has been justified in that the machine has run steadily for the last 15 months at an intensity of 3.5-4.2 $\mu \mathrm{A}$ with high extraction efficiency ( $\sim 70 \%$ in fast burst mode) and a good duty cycle. Down time due to faul ts has been $2 \%$ for the first eight months of 1978.

${ }^{3} \mathrm{He}^{2+}$ ions were accelerated to the full energy of $910 \mathrm{MeV}$ in May this year after a shutdown to install the the coaxial line extension between Dee and Rotco which lowers the frequency range appropriately; a beam of $0.7\lfloor$ A was delivered to 1 solde. The acceleration of ${ }^{3} \mathrm{He}^{2+}$ ions will be a regular feature of the SC operating schedule and, as will be discussed in section $4,{ }^{12} \mathrm{C}^{4+}$ and other ions should also be available next year.

A second change in the facilities at the SC was made during this shutdown - the introduction of a semiautomatic beam switching arrangement between the extracted beam line to Isolde and the external target station. This requires the mechanical movement of magnets with the associated manipulation of vacuum flanges, etc. The new arrangement allows the change-over to be accomplished with very little radiation dose to the personnel involved and increases the time available for physics.

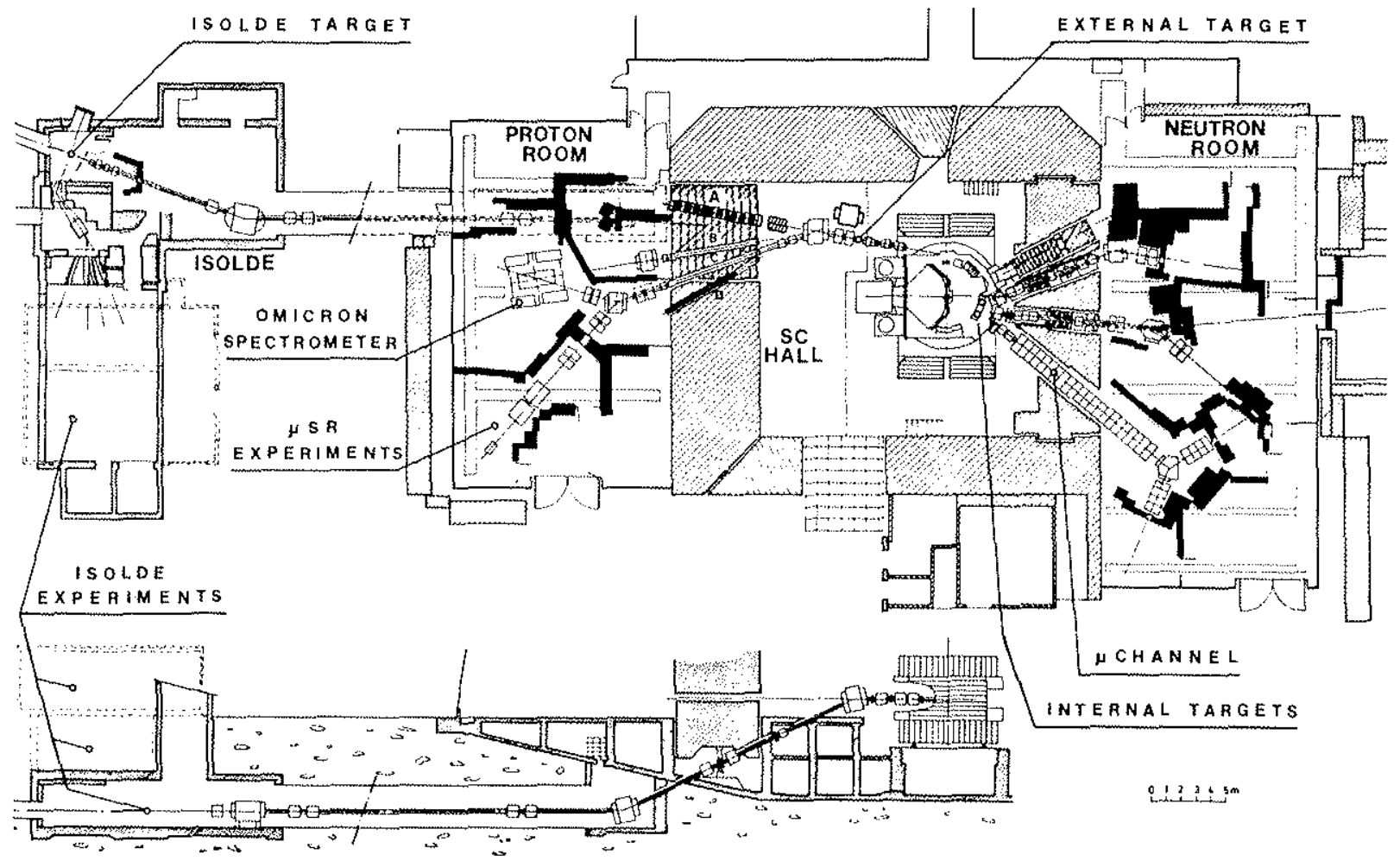

Beams in the "Neutron" Room are derived from internal targets.

Beams in the "Proton" Room are derived from an external target in the SC Hall.

Alternatively the external primary beam is taken underground to the Isolde target.

Fig. 1. SC Layout, Summer 1978.

*) CERN, CH 1211 Geneva 23, Switzerland.
**) For accounts of the situation in 1975 and of improvements to early 1977 see references 1) and 2). 


\section{Present Performance}

\subsection{Experimental Programme}

The accelerator was run for physics in the years 1975,76 and 77 for 2757,4141 and 5779 hours respectively, reflecting the gradual improvement in reliability. There is a very active experimental programe with 18 experiments taking data in the 1 ast 12 months while about 80 experiments were performed at the Isolde facility; over 200 physicists from more than 40 institutes work on experiments at the SC of whom half are at Isolde. The Isolde facility has been increasing its share of the available beam time and now receives an allocation of 25 eight hour shifts per month, one third of the total time scheduled.

The SC also provides parasitic beams for testing equipment used in experiments at the CERN $400 \mathrm{GeV}$ Proton Synchrotron (SPS).

\subsection{Ion Source}

The ion source works well and filament lifetimes of 120 hours are now normal for proton acceleration; this goal had been set. as it allows most filament changes (taking 1-2 hours) to be made during scheduled bean changes or maintenance periods. For ${ }^{3} \mathrm{He}^{2+}$ the filament lifetime is considerably shorter ( 20 hours) because of the higher arc current required.

\subsection{Rotating Condensors (Rotcos)}

A variety of faults have been encountered with the rotating condensors and there has been continuous repair, modification and improvement for the whole $3 \frac{1}{2}$ years since the machine was turned on aga in after SCIP.

The Rotco problems have continued to be mainly mechanical in origin; the most serious was the failure in Rotco 1 of the ceramic bushing protecting the ballbearings - as had happened to Rotco 2 in $1976^{2}$ ). Fortunately the fault was found while the Rotco was being overhauled and upgraded.

one of the items which were known to be critical is the capacitive bridge, which protects the rotor bearings against RF current. A new method of setting the bridge, in which the attenuation is measured in situ, has been developed.

A result of this more precise measurement was to expose the influence of the RF behaviour of elastomer 0urings at electrically critical places near the bridge. Proper tuning could only be achieved after replacement of the Viton rings by silicone ones, which have a much lower loss factor. It is believed that a proper balancing of the bridge, resulting in typically $>60 \mathrm{~dB}$ attenuation at the low frequency end (where the RF current feeding the bridge is highest) constitutes one of the important factors permitting a bearing lifetime in excess of six months of normal operation.

Electrical performance was limited by the mean power rating of two elements. The ceramic blocking capacitors, which are connected in series to the compensating coils and the vacuum capacitors with which the frequency limits are varied. Blocking capacitors, watercooled on both sides have been fitted (so far only to Rotco 2). The vacuum capacitors whose current rating was not adequate to use their full variation range, have now been replaced (so far on Rotco 1) by a type which is water-cooled on both sides, in particular on the bellows.

The result of the improvements so far made is that Rotco 2 is now used during nomal operation at an RF duty cycle of $1: 2$ (i.e. with the RF pulsed once in every two possible cycles) and at $20 \mathrm{kV}$ (instead of the $30 \mathrm{kV}$ originally specified). This is sufficient to accelerate 4 ;A of protons with a sparking rate often down to one per hour. Rotco 1 has not yet been improved to the same extent, though this is planned, and its rated performance is $1: 2$ at $18 \mathrm{kV}$.

\subsection{Internal targets and Bean Sharing ${ }^{3}$ )}

The accelerated bean may strike an internal target to generate secondary particles for use in the Neutron Roon or it may be extracted and sent either towards an external target generating Proton Room beams, or towards the Isolde target. To satisfy the users' demand for bean time a sharing mode of operation is frequently used in which an internal target intercepts a fraction of the accelerated beam, the rest being extracted. The sharing ratio between internal target and extraction is adjusted by altering the axial position of the target. Beam sharing is worth while since the $4 \mathrm{HA}$ beam is high enough ti provide adequate beams for both users simultaneously.

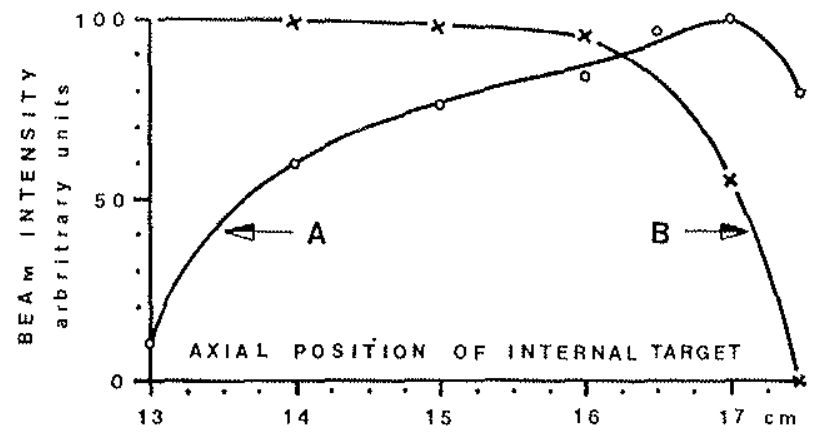

Fig. 2. Secondary bearn from internal target $(A)$, and extracted beam (B) vs internal target position.

Fig. 2 shows a typical example of the beam intensi. ty received by two users as the axial position of the target is varied. The emittance of the extracted beam is increased by sharing and so is not desirable when a minimum external target spot is required.

\subsection{Duty Cycle}

Two methods of stretching the beam in time are avai able: the first is the Cee system, in which the Cee continues the acceleration after the Dee programe is cut off. This gives macro duty cycles of $>50 \%$, but as it is an accelerating system, there is still RF structure in the beam, and for this reason it has proved to be much less popular among users than the alternative method. In addition the Cee frequency and voltage programe are somewhat delicate to set up and for the machine operator to keep optinized.

The pulsed field coil or kim coil is more popular because the RF picro-structure is eliminated and because it is easier to set up. The bean is stacked at a radius just prior to extraction when the Dee programme is cut off. The stacked beam is then driven away from or towards the regenerator (which causes it to be extracted) by means of the field bump caused by an appropriately shaped current pulse in the Kim coil. Overall duty facm tors between $50 \%$ and $80 \%$ have been reported by users.

It was noted in 2.4 above that the beam is often shared. The setting up of this sharing involves not only 
the adjustment of the intensities, but also the sesta blisfinent of good duty cycle conditions for both wars With both the cee and the kin coll systems this opera. tion is somemat delicate, but it has been fomd that both users can be satisfied simutaneously. In the case of xim coil operation, a small residual "fast burst" spike appears in both beans.

\subsection{Extraction Efficiency}

The SCIP design goal of an improvenent of a factor 10 in the efficiency of extracting a beam has been amply achieved. With the new extraction systen a theoretical maximum of $73 \%$ is possible. Our best measurenents approach this value, but the errors are quite large ( $10 \%$ ) since (a) there us no measurement of the bean at large radius just prior to extraction: it is measured at low radius using a Faraday cup and (b) after extraction it is measured using a secondary emission monitor calibrated by activation. The extraction efficiency then the Kin coll is in use is slightly lower than in fast burst operation.

\section{Recent Developments}

\section{1. ${ }^{3} \mathrm{He}^{2+}$ Acceleration $\left.{ }^{4}, 5,6\right)$}

The acceleration of ${ }^{3} \mathrm{He}^{2+}$ requires a frequency band of $20.0 \mathrm{MAz}$ to $13.9 \mathrm{WHz}$ whereas for protons the range is $30.1 \mathrm{MHz}$ to $16.8 \mathrm{MHz}$. This change in frequency band is achieved by introducing a transmission line between the Dee and the Rotco, consisting of a flat tank $120 \mathrm{~cm}$ long, $136 \mathrm{~cm}$ wide and $36 \mathrm{~cm}$ high, containing a similarly sham ped inner conductor (see fig. 3).

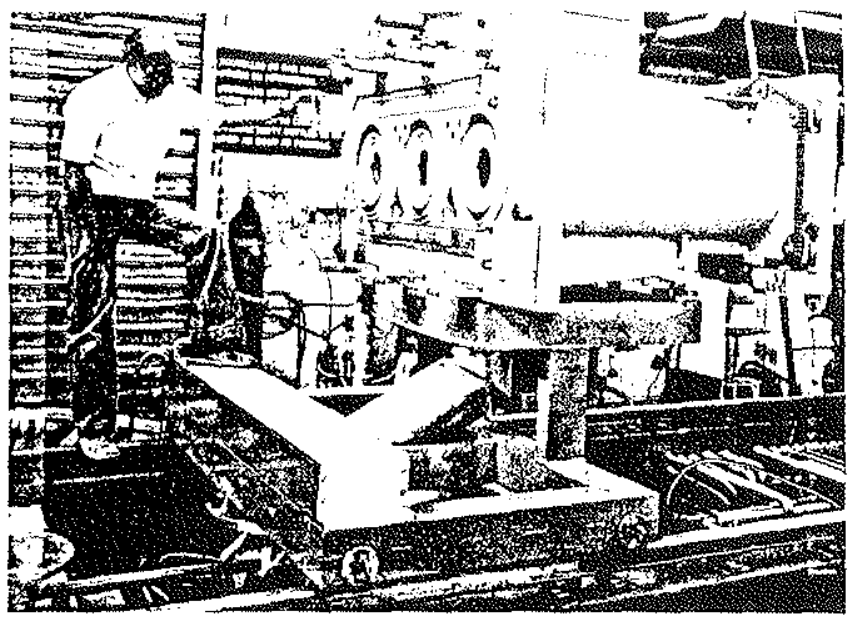

Fig. 3. RF extension line.

The tank is evacuated and both it and the inner conductor are water-cooled. The introduction of this tank is facilitated by the fact that the Rotco and the RF generator can be moved away from the cyclotron on rails, thus opening up the necessary $120 \mathrm{cmlgap}$. An additiona? $3500 \mathrm{l} / \mathrm{s}$ turbo-pump was added to evacuate the Rotco in its "Hezt position.

The mechanical installation of the additional transmission line can be achieved in less than one shift; pumping and RF conditioning take a further 4 or 5 shifts. For this reason it is most convenient to schedule blocks of time with a ${ }^{3} \mathrm{He}^{2}$ t beanl several times each year.

The ion source used for protons can be used for ${ }^{3}$ Het ions with no modifications other than the recomection of the gas bottle. However, a higher arc current is repired with a consequent reduction of filament lifetimes. In iay 1978 a 0.7 W bean was delivered to isolde and it is anticipated that this current will increase by at least a factor 2 in subsequent runs.

The first results of experiments with the new beam are very encouraging. In the case of certain Isolde experiments the use of ${ }^{3} \mathrm{He}^{24}$ ions of $910 \mathrm{MeV}$ resulted in a 20 -fold increase in counting rate compared to the use of 600 MeV protons.

\subsection{Bean Switching}

The extracted beam is used by Isolde or for production of secondaries for experinents in the Proton Hall. Because of lack of space in the Cyclotron Hall, the change-over between these different modes required the replacenent of the first 20 ton vertical bending magnet in the Isolde beam line by the external production target and the first lens doublet of the secondary heam; implying a series of other jobs ranging from the making of vacuum connections to the shifting of TV cameras. The schedule requires this change-over to be made at least once a week. Coupled with the increased extracted bean intensity high radiation doses to the specialised per. sonnel involved was becoming a serious problem.

The process of change-over has therefore been mechanized during the spring shutdown. The whole operation can now be done in 2 to 3 hours with minimal intervention by an operator (rather than a mechanic) and the doses received are much reduced. In addition, there is no longer any need for a radiation cooling shift so that the scheduling has become more flexible. A photograph of the chariot assembly is shown in fig. 4.

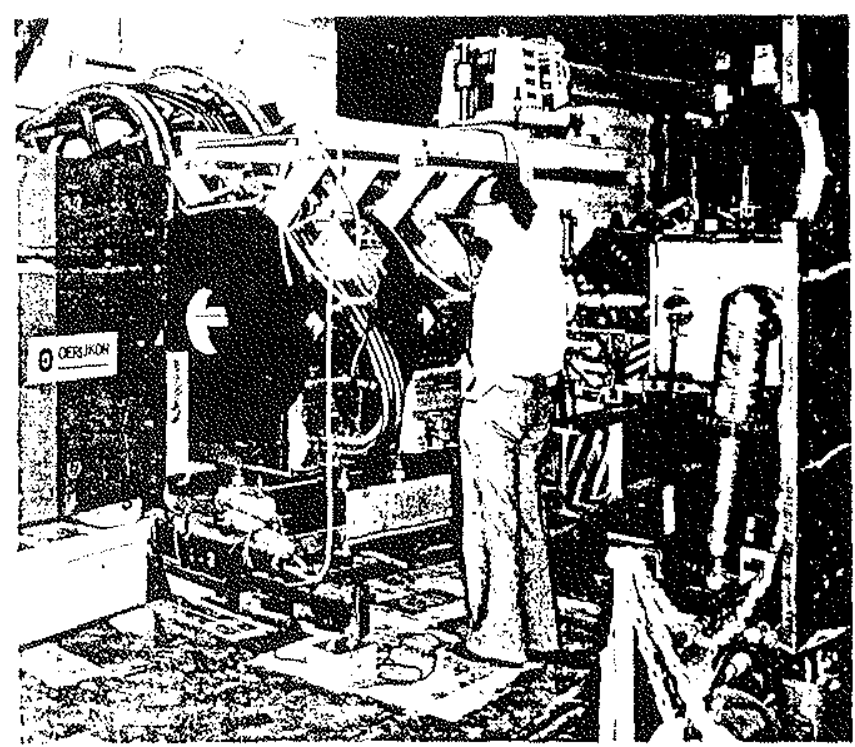

Fig. 4. External target/beam transport chariot.

\subsection{Extracted Beam Quality}

At the same time as the mechanization mentioned above all bindows were removed from the isolde beam line and retractable alumina screens and secondary emission monitors were installed. Previously the SC vacum was separated from the Isolde bean line by two windows and an air gap, and in addition two secondary emission monitors were permanently installed in the extracted beam- 
line. The scattering caused by all this material in the beam increased the enittance considerably.

The effect of these changes is an improvenent in the spot produced at the Isolde target and a reduction of the transmission losses, al though not by as large a factor as expected. The reason for this is probably that the beam from the cyclotron has two components which could not be observed hitherto. The photograph in fig. 5 shows the spot on an alumina screen placed in after the extraction port: the lenses are all off, and the SC is operating in fast burst. The main part of the beam has an octupole shape provoked by passage through the extraction channel and the SC fringe field, but there is aiso another component. The origin of this double beam is not yet certain but it is thought to be due to scattering at the septum of the extraction channel.

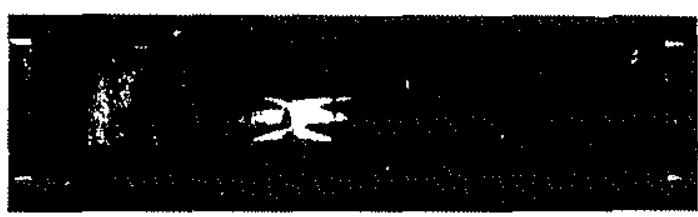

Fig. 5. Extracted beam spot showing two components.

\section{Future Developments}

A shopping list of possible SC developments") was presented to the Workshop on the Role of CERN in European Intermediate Energy Physics held in September 1977. Two SC projects were given importance ${ }^{8}$ ) - firstly to accelerate ${ }^{12} C^{4+}$ ions 9,30 ) (and a limited number of other ions having $Q / A \cong 1 / 3$ ) for use by Isolde and for counter experiments, and, secondly to construct an "Arizona" polarised $\mu^{+}$beam for the active solid state physics and chemistry programmes using ${ }^{\prime} S R^{11}{ }^{1}$.

Competition from other CERN projects prevented authorization of the Arizona beam, but the possibilities of providing a reasonable beam of polarised $\mu^{+}$from an internal target are being looked into.

\section{1. ${ }^{12} \mathrm{C}^{4+}$ Acceleration}

The present SC source (hooded arc, hot cathode) can produce multiply charged light ions with no mudification. This was proven on the source test stand and by accelerating the light ions on the third harmonic mode up to snall radii inside the $\left.S^{12}\right)$. The currents achieved were small (e.g. about $10 \mathrm{nA}$ of ${ }^{20} \mathrm{Ne}^{4+}$ ) but the loss due to charge exchange was lower than expected from the accepted tank pressure of 1 ;Tor ${ }^{13}$ ). New neasurements showed that, after several weeks punping, the pressure is around 2-3 $\left(0^{-7} \operatorname{Torr}^{14}\right)$, which fits reasonably with the observed beam loss. This stimulated us to further examine the prospects of light ion acceleration in the $\mathrm{SC}$.

ro produce a sufficient current of $12 \mathrm{C}^{4+}$ and other ions with a similar charge to mass ratio $(Q / A)$, the arc current in the source will need to be increased considerably:s). The resulting increase in power dissipation makes water-cooling of the source head essential. Fortunately calculations on the first orbit of the ions after extraction from the source showed that there is enough space available to house a cooled source head and to incorporate other new features (e.g. connection of the anti-cathode to a fixed potential and a gas-distributor to equalize the pressure along the plasma cnamberl. A new central geometry will also be constructed to ensure good centring and focusing of the ions in the centre of the SC.

The acceleration of ${ }^{12} \mathrm{C}^{4+}$ and other light ions with $Q / A$ ratio near $1 / 3$, requires the $R F$ system to cover a frequency range of $11 \mathrm{MHz}$ to $8 \mathrm{MHz}$ if acceleration is done on the fundamental frequency. This frequency is so far below that for protons $(30.1 \mathrm{MHz}$ to $16.8 \mathrm{iHz}$ ) that it was not expected that a suitable matching between Dee and Rotco could be found. For this reason, initially, possibility of accelerating ions on the third harmonic of their resonant frequency was examined ${ }^{16}$ ).

The complications of applying this method were such that the feasibility of working on the first harmonic was re-examined, both theoretically and experimentally, using the $1 / 5$ scale model of the SC RF system ${ }^{27}$ ). A very economical solution has been found in which the concentric inner conductor of the ${ }^{3} \mathrm{He}^{2+}$ extension line is replaced by a pair of parallel tubes; the outer conductor, which is also the vacuum tank, support trolley and pumping system are unchanged.

The project was approved in June this year and we expect to do the first trials in one year from now.

Expected performances are given in the table below.

\begin{tabular}{|c|c|c|c|}
\hline Ion & $\begin{array}{l}\text { Ene } \\
\mathrm{MeV}\end{array}$ & $\begin{array}{l}g y \\
\frac{\mathrm{MeV}}{\mathrm{amu}}\end{array}$ & $\begin{array}{c}\text { Extracted intensity, } \\
\text { ions } / \mathrm{s}\end{array}$ \\
\hline${ }^{14} \mathrm{~N}^{5+}$ & 1372 & 98 & factor 100 lower than ${ }^{12} \mathrm{C}^{4+}$ \\
\hline${ }^{12} \mathrm{C}^{4+}$ & 1032 & 86 & $\left\{\begin{array}{l}\text { pessimistic }: 5.10^{10} \text { ions } / \mathrm{s} \\
\text { optimistic }: 10^{12} \text { ions } / \mathrm{s}\end{array}\right.$ \\
\hline${ }^{19} \mathrm{~F}^{6+}$ & 1463 & 77 & factor 100 lower than ${ }^{12} \mathrm{C}^{4+}$ \\
\hline $160^{5+}$ & $12\} 6$ & 76 & factor 10 lower than ${ }^{12} \mathrm{C}^{4+}$ \\
\hline
\end{tabular}

Contributors to the ${ }^{12} \mathrm{C}^{4+}$ project proposal are: A. Fiebig, R. Galiana, R. Giannini, C.E. Hill, R. Hohbach J.H.B. Madsen, A. Susini, S. Talas and N. Vogt-Nilsen.

\section{References}

1) H. Beger, F. Blythe, G. Le Dallic, H. Lustig, E.G. Michaelis, N. Vogt-Nilsen, Proc. Seventh Int. Cyc. Conf., p. 49, Zürich (1975).

2) B.W. Allardyce, H. Beger, R. Galiana, R. Gjannini, E.G. Michaelis, S. Talas, IEEE Trans. Nuc. Sci., NS-24, no 3, p 1631 (1977).

3) G. Le Dallic, P. Mandrillon, A. Zanada, SC Techn. Dev. Note No. $55,22.12 .77$.

4) A. Fiebig, PS-CD/131,30.7.1976

5) R. Giannini, PS-CD/183, 15.10.1976.

6) R. Galiana, SC Techn. Dev. Note no.44, 1.3.1977.

7) B.W. Allardyce, J.H.B. Madsen, P.H. Standley, PS-CD/77-24, 22.7.1977.

8) R. Klapisch, PS-CD/77-50 (rev.)

9) B.W. Al Jardyce, A. Fiebig, R. Galiana, R. Giannini, J.H.B. Madsen, P.H. Standley, N. Vogt-Nilsen, PS-CD/77-36, 20.9.1977.

10) J.H.B. Madsen, SCC/78-14, 26.4.1978.

11) B.W. Allarcyce, SCC/78-13, 26.4.1978.

12) R. Galiana, SC Techn. Dev. Note no. 52, 24.10.1977.

13) R. Giannini, SC Techn. Dev. Note no.54, 7.12.1977.

14) A. Blin, B. Gay, SC Techn. Dev. Note no. $57,5.10 .1977$.

15) R. Galiana, PS-CO/Note 78-5 rev., 13.4.1978.

16) R. Giannini, P. Mandrillon, Proc. 7 th int. Cyc. Conf, p. 609, Zürich (1975).

17) R. Hohbach, PS CD/Note 78-10, 17.7.1978. 